\title{
Density Functional Theory; a New Route for Structural and Thermodynamic Parameters Calculations
}

\author{
A.M. Abdelghany ${ }^{a}$, M.S. Meikhail ${ }^{b}$, W. Awad ${ }^{b}$ \\ a Spectroscopy Department, Physics Division, National Research Centre, 33 Elbehouth St., Dokki, 12311, \\ Giza, Egypt.
}

${ }^{\mathrm{b}}$ Physics Department, Faculty of Science, Mansoura University, Mansoura, 35516, Egypt.

E-mail: a.m_abdelghany@yahoo.com

\begin{abstract}
Structural and thermodynamic parameters of the studied virgin polymeric matrices poly $\varepsilon$-caprolactone $(\mathrm{PCL})$, Chitosan (Chi) and their polymer blend were investigated using theoretical density functional theory (DFT). Thermodynamic functions were calculated based on vibrational frequencies and optimized geometrical coordinates in the temperature range $25-1000{ }^{\circ} \mathrm{K}$ which cannot be amended easily. Calculated free energy was found to be convex in terms of extensive variable and concave in terms of intensive ones.
\end{abstract}

Keywords: Density functional theory (DFT); Thermodynamic parameters; Free energy; Entropy; Specific heat.

\section{Introduction}

Physical and thermodynamic parameters of solids are attributable to their structural arrangement and also to the internal energy of their complexes. Experimental measurement of such structural parameters are limited due to the expenses and facilities that can give results with accurate precision. Polymer blends are composed of two or more organic polymer matrices. Due to the unsatisfactory thermal, mechanical and electric properties, there is a need to functionalize them in an attempt to improve their properties to extend their potential applications [1, 2]. Chitosan (Chi) is obtained from chitin, the second most abundant polysaccharide in nature, which is found in the exoskeletons of crustaceans such as crab and shrimp [3]. Chitin comprises of a linear chain of Nacetyl D-glucosamine molecules. Deacetylaton of chitin to various degrees (typically 60-95\%) in hot concentrated alkali removes the acetyl groups and forms chitosan. Therefore, chitosan may be considered as a copolymer of D-glucosamine and $\mathrm{N}$-acetyl Dglycosamine residues where the relative ratio of $\mathrm{D}$-glucosamine to $\mathrm{N}$-acetyl-Dglucosamine represents the degree of deacetylation of chitosan. The chemical structure of chitosan has been investigated by Fourier Transform Infrared Spectroscopy (FTIR) in which functional groups of a molecule absorb infrared radiation at specific wavenumbers. FTIR of chitosan shows the amine and hydroxyl functional groups which show overlapping peaks in 3300-3500 cm-1 [3-5]. The degradation rate is dependent on several factors including $\mathrm{MW}, \mathrm{DD}$, local $\mathrm{pH}$, temperature, structural stability and homogeneity of source [6-8].Poly( $\varepsilon$-caprolactone) $(\mathrm{PCL})$ is one of the most flexible and easy to process polymers, but also one of the slowest degrading ones [9]. This could be achieved by mixing chitosan and PCL for their unique contribution to the necessary biomechanical properties. However, chemical reactions such as copolymerization or crosslinking between chitosan and PCL may compromise their individual properties. Therefore, an ideal option is to make the polymeric components co-exist by mixing them physically. Physical mixing can be done by dissolution in a common solvent. However, there is a lack of common solvent for chitosan and PCL as they are hydrophilic and hydrophobic by nature, respectively. Chitosan and PCL mixtures present a model system for studying composites of two semi-crystalline polymers that differ in charge distribution, as chitosan is cationic in nature and PCL is uncharged.Blending two polymers is an approach to develop new biomaterials exhibiting combinations of properties that could not be obtained by individual polymers and to develop a new composite biomaterial that can be fabricated into thin films with alterable mechanical [10], biological and degradation properties for the regeneration of a variety of soft tissues [11]. The hypothesis of this work is that chitosan and $\mathrm{PCL}$ are versatile polymers with mutually complementary biomechanical properties and combining them would result in a unique biomaterial that can be fabricated into three-dimensional constructs with tunable physicochemical and biomechanical properties for a wide range of tissue engineering applications. As a modern technique of physics, Computational molecular modeling has greatly accelerated success in the determination of structural parameters, vibrational frequencies, and also 
thermodynamic properties of complexes. It is started in the last several decades and improvements in the computing power help physicist to predict thermodynamics of polymeric systems which is difficult to be measured experimentally. Determination of the thermodynamic quantities from experiments is always constrained by the particular technique used, and it may difficult to compare quantities derived from different techniques.

\section{Materials and Methods}

Selected polymeric material including poly $\varepsilon$-caprolactone (PCL), Chitosan (Chi) and their semi-natural polymer blend with a huge number of applications were studied theoretically for their atomic arrangement, interaction and their thermodynamic parameters. The efficient density functional theory (DFT) was applied to determine the structural parameters and thermodynamic parameters.

Studied thermodynamic functions of selected materials were calculated on the base of optimized geometrical coordinates for temperature interval between 25-1000 K. DFT/B3LYP method was applied for the calculations of the equilibrium geometrical and thermodynamic parameters of chitosan, $\mathrm{PCL}$ and their blend.

\section{Formalism of thermodynamics quantities}

For a free energy $F$, the condition of stability must be slightly amended, as it was generated from the energy using a Legendre transform. For an extensive variable $X$, the stability condition is identical to that of the energy,

$$
\frac{\partial^{2} F}{\partial X^{2}} \geq o,
$$

and the free energy is convex in X. However, for intensive variables $\mathrm{x}$, the free energy is concave in $\mathrm{x}$ :

$$
\frac{\partial^{2} F}{\partial x^{2}} \leq o
$$

The first derivatives of the free energies have been previously given a physical meaning. The second derivatives also have physical meaning for certain free energies, and in these contexts they are known as "susceptibilities". Two important susceptibilities are the heat capacity $\mathrm{Cp}$, which is an extensive measure of the heat flow necessary to change temperature,

$$
C \equiv \frac{\delta Q}{d T}=T \frac{d S}{d T}
$$

and the bulk modulus $K$, an intensive measure of the change in pressure upon expansion/contraction of the system,

$$
K \equiv-V \frac{d P}{d V}
$$

Both equations $(3,4)$ assume a particular process is being carried out, the former that temperature is changing and the later that volume is changing, but knowledge of the other constrained variable are necessary to convert these into proper thermodynamic quantities. It is usually assumed that particle 
number is fixed, so the heat capacity may be evaluated at fixed pressure $\mathrm{Cp}$ or fixed volume $\mathrm{CV}$, and the bulk modulus may be evaluated at fixed temperature KT or fixed entropy KS. Each quantity suggests a natural ensemble,

$$
\begin{aligned}
& K_{T}=V \frac{\partial^{2} A}{\partial V^{2}} \mid T,\left\{N_{i}\right\} \\
& K_{S}=V \frac{\partial^{2} U}{\partial V^{2}} \mid S,\left\{N_{i}\right\} \\
& C_{V}=-T \frac{\partial^{2} A}{\partial T^{2}} \mid V,\left\{N_{i}\right\} \\
& C_{P}=-T \frac{\partial^{2} G}{\partial T^{2}} \mid P,\left\{N_{i}\right\}
\end{aligned}
$$

The stability requires all four of these quantities to be positive, and the specific heat and bulk modulus must always be non-negative. 


\section{Results and Discussion}

DFT route can be used to perform calculations without fitted parameters, so, it is a power tool to examine the polymeric materials. It predicts the structures stability in the infinite time and volume. DFT route also can provide energy optimization of the three dimensional network of solid material unit structure.

Optimized three dimensional polymeric network of the studied solid material Chi, PCL and their suggested interaction counterpart can be shown in Figure $(1, a-c)$. Calculated values of molar heat capacity $C_{p}$ Gibbs reduced free energy $\Phi$, entropy $S$ and enthalpy increment $H(T)-H(0)$ corresponding to this optimized structures are listed in Tables (1-3) for Chitosan, $\mathrm{PCL}$, and their blend respectively.

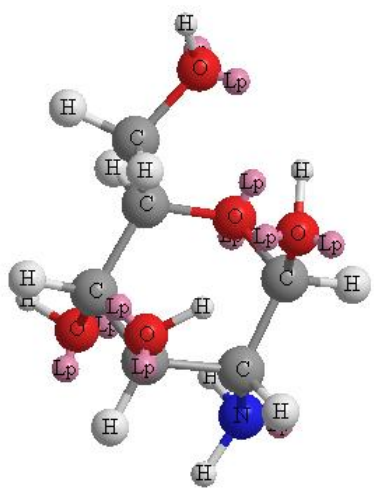

(a) Optimized structure of Chitosan

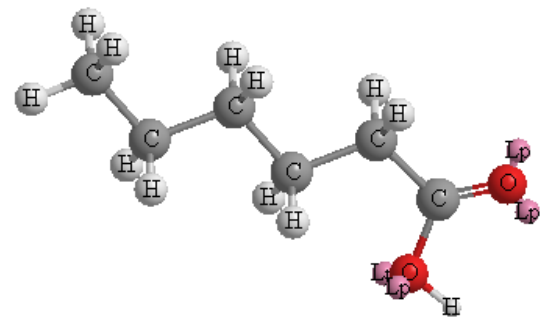

(b) Optimized structure of PCL

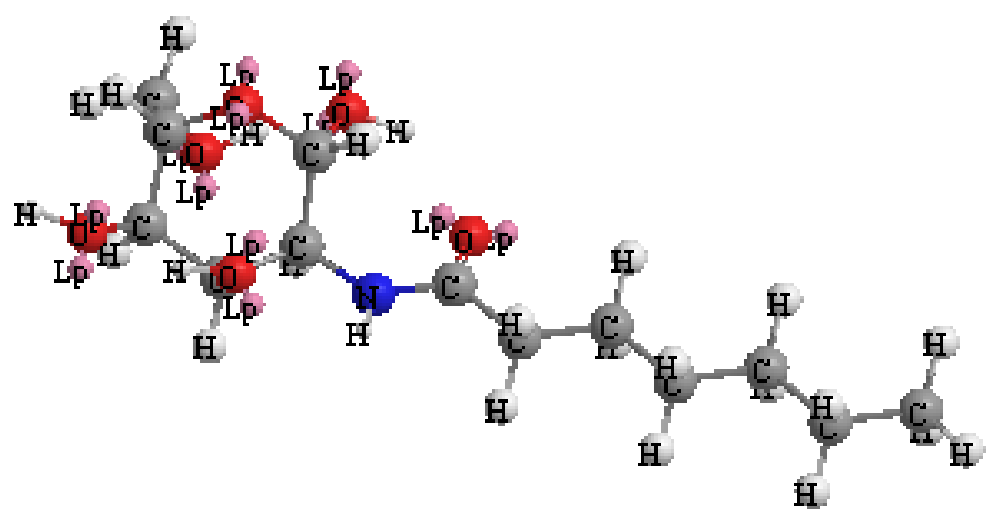

(c) Optimized structure of PCL/Chi blend

Figure 1 Optimized structure of studied polymeric materials 
Table (1): The thermodynamics properties of chitosan.

\begin{tabular}{|c|c|c|c|c|}
\hline$T(K)$ & $\begin{array}{l}\mathbf{C p} \\
\left(J . \mathrm{mol}^{-1} \mathrm{~K}^{-1}\right)\end{array}$ & $\begin{array}{l}\mathbf{S} \\
\left(\mathrm{J} \cdot \mathrm{mol}^{-1} \cdot \mathrm{K}^{-1}\right)\end{array}$ & $\begin{array}{l}\boldsymbol{\phi} \\
\left(\mathrm{J} \cdot \mathrm{mol}^{-1} \cdot \mathrm{K}^{-1}\right)\end{array}$ & $\begin{array}{l}\mathbf{H}(\mathbf{T})-\mathbf{H}(\mathbf{O}) \\
\left(\mathrm{kJ} . \mathrm{mol}^{-1}\right)\end{array}$ \\
\hline 25 & 21.743 & 71.701 & 354.108 & 355.901 \\
\hline 50 & 38.125 & 92.024 & 352.056 & 356.657 \\
\hline 75 & 51.906 & 110.132 & 349.526 & 357.786 \\
\hline 100 & 64.917 & 126.858 & 346.561 & 359.247 \\
\hline 125 & 77.504 & 142.703 & 343.19 & 361.028 \\
\hline 150 & 89.715 & 157.918 & 339.431 & 363.119 \\
\hline 175 & 101.661 & 172.647 & 335.298 & 365.511 \\
\hline 200 & 113.47 & 186.994 & 330.802 & 368.201 \\
\hline 225 & 125.222 & 201.038 & 325.951 & 371.184 \\
\hline 250 & 136.932 & 214.838 & 320.752 & 374.461 \\
\hline 275 & 148.557 & 228.435 & 315.211 & 378.03 \\
\hline 298.15 & 159.182 & 240.867 & 309.778 & 381.593 \\
\hline 300 & 160.023 & 241.854 & 309.332 & 381.888 \\
\hline 325 & 171.246 & 255.108 & 303.119 & 386.029 \\
\hline 350 & 182.146 & 268.2 & 296.578 & 390.448 \\
\hline 375 & 192.659 & 281.127 & 289.711 & 395.134 \\
\hline 400 & 202.736 & 293.885 & 282.523 & 400.077 \\
\hline 425 & 212.35 & 306.467 & 275.018 & 405.266 \\
\hline 450 & 221.487 & 318.866 & 267.201 & 410.69 \\
\hline 475 & 230.147 & 331.075 & 259.076 & 416.337 \\
\hline 500 & 238.341 & 343.09 & 250.649 & 422.194 \\
\hline 525 & 246.085 & 354.908 & 241.923 & 428.25 \\
\hline 550 & 253.402 & 366.527 & 232.905 & 434.495 \\
\hline 575 & 260.314 & 377.945 & 223.599 & 440.917 \\
\hline 600 & 266.848 & 389.163 & 214.009 & 447.507 \\
\hline 625 & 273.029 & 400.183 & 204.142 & 454.256 \\
\hline
\end{tabular}




\begin{tabular}{lllll}
650 & 278.88 & 411.006 & 194.002 & 461.156 \\
675 & 284.427 & 421.636 & 183.594 & 468.198 \\
700 & 289.689 & 432.076 & 172.922 & 475.375 \\
725 & 294.689 & 442.329 & 161.991 & 482.68 \\
750 & 299.444 & 452.401 & 150.807 & 490.107 \\
775 & 303.97 & 462.294 & 139.373 & 497.65 \\
800 & 308.285 & 472.013 & 127.694 & 505.304 \\
825 & 312.401 & 481.563 & 115.774 & 513.063 \\
850 & 316.332 & 490.948 & 103.617 & 520.923 \\
875 & 320.09 & 500.172 & 91.227 & 528.878 \\
900 & 323.684 & 509.24 & 78.609 & 536.926 \\
925 & 327.124 & 518.156 & 65.767 & 545.061 \\
950 & 330.42 & 526.924 & 52.703 & 553.281 \\
975 & 333.579 & 535.548 & 39.422 & 561.581 \\
1000 & 336.608 & 544.032 & 25.927 & 569.958 \\
\hline
\end{tabular}


Table (2): The thermodynamics properties of PCL.

\begin{tabular}{|c|c|c|c|c|}
\hline$T(K)$ & $\begin{array}{l}\mathbf{C p} \\
\left(\mathrm{J} . \mathrm{mol}^{-1} \mathrm{~K}^{-1}\right)\end{array}$ & $\begin{array}{l}\mathbf{S} \\
\left(\mathrm{J} \cdot \mathrm{mol}^{-1} \cdot \mathrm{K}^{-1}\right)\end{array}$ & $\begin{array}{l}\boldsymbol{\phi} \\
\left(\mathrm{J} \cdot \mathrm{mol}^{-1} \cdot \mathrm{K}^{-1}\right)\end{array}$ & $\begin{array}{l}\mathbf{H}(\mathrm{T})-\mathbf{H}(\mathbf{O}) \\
\left(\mathrm{kJ} \cdot \mathrm{mol}^{-1}\right)\end{array}$ \\
\hline 25 & 8.72 & 49.831 & 108.212 & 109.458 \\
\hline 50 & 11.211 & 56.627 & 106.875 & 109.707 \\
\hline 75 & 13.667 & 61.642 & 105.395 & 110.018 \\
\hline 100 & 15.928 & 65.889 & 103.799 & 110.388 \\
\hline 125 & 18.032 & 69.671 & 102.104 & 110.813 \\
\hline 150 & 20.079 & 73.14 & 100.318 & 111.289 \\
\hline 175 & 22.151 & 76.39 & 98.449 & 111.817 \\
\hline 200 & 24.3 & 79.487 & 96.5 & 112.398 \\
\hline 225 & 26.551 & 82.478 & 94.476 & 113.033 \\
\hline 250 & 28.906 & 85.396 & 92.377 & 113.726 \\
\hline 275 & 31.35 & 88.265 & 90.206 & 114.479 \\
\hline 298.15 & 33.669 & 90.892 & 88.132 & 115.232 \\
\hline 300 & 33.856 & 91.1 & 87.964 & 115.294 \\
\hline 325 & 36.393 & 93.91 & 85.651 & 116.172 \\
\hline 350 & 38.931 & 96.7 & 83.269 & 117.114 \\
\hline 375 & 41.442 & 99.472 & 80.816 & 118.118 \\
\hline 400 & 43.904 & 102.226 & 78.295 & 119.185 \\
\hline 425 & 46.302 & 104.96 & 75.705 & 120.313 \\
\hline 450 & 48.623 & 107.672 & 73.047 & 121.5 \\
\hline 475 & 50.861 & 110.361 & 70.322 & 122.744 \\
\hline 500 & 53.012 & 113.025 & 67.53 & 124.042 \\
\hline 525 & 55.075 & 115.662 & 64.671 & 125.393 \\
\hline 550 & 57.052 & 118.27 & 61.747 & 126.795 \\
\hline 575 & 58.946 & 120.848 & 58.758 & 128.245 \\
\hline 600 & 60.758 & 123.396 & 55.704 & 129.742 \\
\hline 625 & 62.494 & 125.911 & 52.588 & 131.283 \\
\hline 650 & 64.156 & 128.395 & 49.409 & 132.866 \\
\hline
\end{tabular}




\begin{tabular}{lllll}
675 & 65.75 & 130.846 & 46.169 & 134.49 \\
700 & 67.277 & 133.265 & 42.867 & 136.153 \\
725 & 68.743 & 135.652 & 39.506 & 137.853 \\
750 & 70.15 & 138.006 & 36.085 & 139.59 \\
775 & 71.501 & 140.329 & 32.606 & 141.36 \\
800 & 72.8 & 142.619 & 29.069 & 143.164 \\
825 & 74.048 & 144.879 & 25.475 & 145 \\
850 & 75.249 & 147.107 & 21.825 & 146.866 \\
875 & 76.404 & 149.305 & 18.12 & 148.762 \\
900 & 77.516 & 151.473 & 14.36 & 150.686 \\
925 & 78.586 & 153.612 & 10.546 & 152.637 \\
950 & 79.616 & 155.721 & 6.68 & 154.615 \\
975 & 80.609 & 157.802 & 2.76 & 156.618 \\
1000 & 81.565 & 159.855 & -1.21 & 158.645 \\
\hline
\end{tabular}


Table (3): The thermodynamics properties of blend.

\begin{tabular}{|c|c|c|c|c|}
\hline$T(K)$ & $\begin{array}{l}\mathbf{C p} \\
\left(\mathrm{J} . \mathrm{mol}^{-1} \mathrm{~K}^{-1}\right)\end{array}$ & $\begin{array}{l}\mathbf{S} \\
\left(\mathrm{J} . \mathrm{mol}^{-1} \cdot \mathrm{K}^{-1}\right)\end{array}$ & $\begin{array}{l}\boldsymbol{\phi} \\
\left(\mathrm{J} . \mathrm{mol}^{-1} \cdot \mathrm{K}^{-1}\right)\end{array}$ & $\begin{array}{l}\mathbf{H}(\mathbf{T})-\mathbf{H}(\mathbf{O}) \\
\left(\mathrm{kJ} . \mathrm{mol}^{-1}\right)\end{array}$ \\
\hline 25 & 11.868 & 59.944 & 241.754 & 243.253 \\
\hline 50 & 18.904 & 70.274 & 240.122 & 243.636 \\
\hline 75 & 26.001 & 79.297 & 238.251 & 244.198 \\
\hline 100 & 32.487 & 87.681 & 236.162 & 244.931 \\
\hline 125 & 38.386 & 95.574 & 233.871 & 245.817 \\
\hline 150 & 43.891 & 103.063 & 231.387 & 246.847 \\
\hline 175 & 49.223 & 110.23 & 228.72 & 248.011 \\
\hline 200 & 54.564 & 117.151 & 225.878 & 249.308 \\
\hline 225 & 60.025 & 123.891 & 222.864 & 250.74 \\
\hline 250 & 65.648 & 130.505 & 219.684 & 252.31 \\
\hline 275 & 71.422 & 137.032 & 216.34 & 254.023 \\
\hline 298.15 & 76.863 & 143.021 & 213.098 & 255.74 \\
\hline 300 & 77.301 & 143.498 & 212.833 & 255.882 \\
\hline 325 & 83.221 & 149.919 & 209.165 & 257.889 \\
\hline 350 & 89.119 & 156.302 & 205.337 & 260.043 \\
\hline 375 & 94.934 & 162.65 & 201.35 & 262.344 \\
\hline 400 & 100.618 & 168.959 & 197.205 & 264.789 \\
\hline 425 & 106.135 & 175.225 & 192.903 & 267.374 \\
\hline 450 & 111.46 & 181.444 & 188.444 & 270.094 \\
\hline 475 & 116.577 & 187.608 & 183.831 & 272.945 \\
\hline 500 & 121.479 & 193.713 & 179.064 & 275.921 \\
\hline 525 & 126.167 & 199.755 & 174.146 & 279.017 \\
\hline 550 & 130.643 & 205.728 & 169.077 & 282.228 \\
\hline 575 & 134.914 & 211.63 & 163.86 & 285.547 \\
\hline 600 & 138.99 & 217.459 & 158.496 & 288.972 \\
\hline 625 & 142.881 & 223.212 & 152.988 & 292.495 \\
\hline 650 & 146.595 & 228.889 & 147.336 & 296.114 \\
\hline
\end{tabular}




\begin{tabular}{lllll}
675 & 150.144 & 234.489 & 141.544 & 299.824 \\
700 & 153.536 & 240.011 & 135.613 & 303.62 \\
725 & 156.783 & 245.456 & 129.544 & 307.499 \\
750 & 159.891 & 250.824 & 123.34 & 311.458 \\
775 & 162.87 & 256.115 & 117.004 & 315.493 \\
800 & 165.726 & 261.332 & 110.535 & 319.601 \\
825 & 168.467 & 266.473 & 103.938 & 323.778 \\
850 & 171.098 & 271.542 & 97.212 & 328.023 \\
875 & 173.627 & 276.538 & 90.361 & 332.332 \\
900 & 176.057 & 281.464 & 83.386 & 336.704 \\
925 & 178.394 & 286.32 & 76.289 & 341.134 \\
950 & 180.642 & 291.107 & 69.071 & 345.622 \\
975 & 182.805 & 295.828 & 61.734 & 350.166 \\
1000 & 184.888 & 300.482 & 54.28 & 354.762 \\
\hline
\end{tabular}


Figure (2) reveals relation between temperature and entropy of chitosan, PCL and their blend in the temperature range $25-1000 \mathrm{~K}$. Such range of temperature cannot be reachable or obtainable in any experimental device.

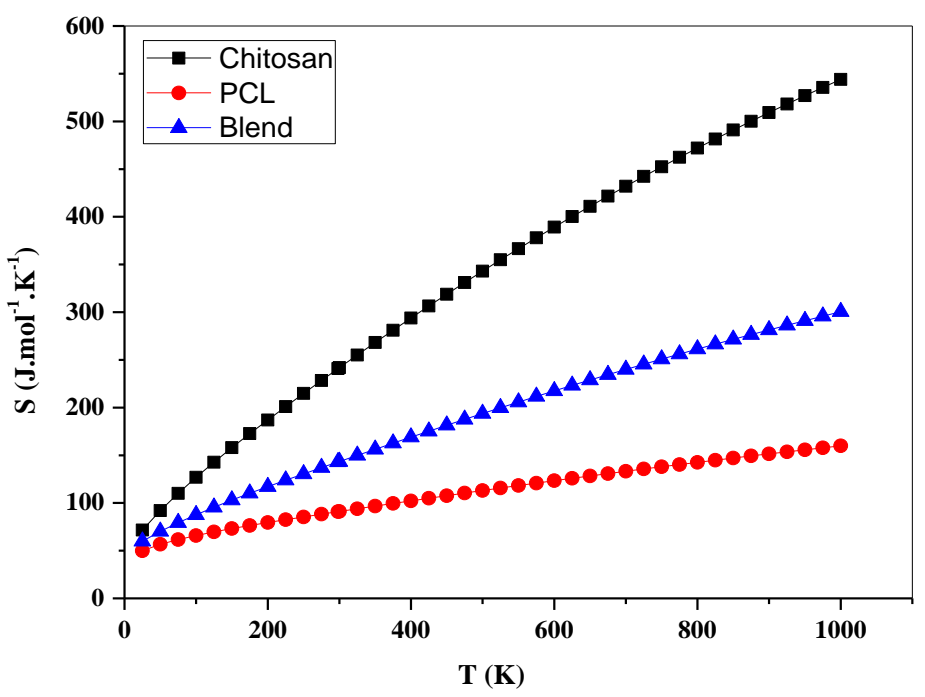

Figure (2) temperature versus entropy of the studied samples

It was noticed from this figure that Chitosan has the largest entropy while the PCL has the lowest and the blend have intermediate values between both partners. The entropy for all of them increased by increasing the temperature in the range from $25-1000 \mathrm{~K}$. It is evident that mixing Chi and PCL the configuration of both of them changed to a new configuration.

Figure (3) shows the relation between both temperature and calculated specific heat capacity of the studied materials. It was observed that the heat capacity of studied polymeric materials was gradually increase in the temperature range from $25-1000 \mathrm{~K}$, the rate of increase was found to be different for each partner. It is noted that Chi has the highest rate of increase over PCL while the blend shows an intermediate values between both polymers in full temperature range indicating some type of interaction and complexation between both Chi and PCL.

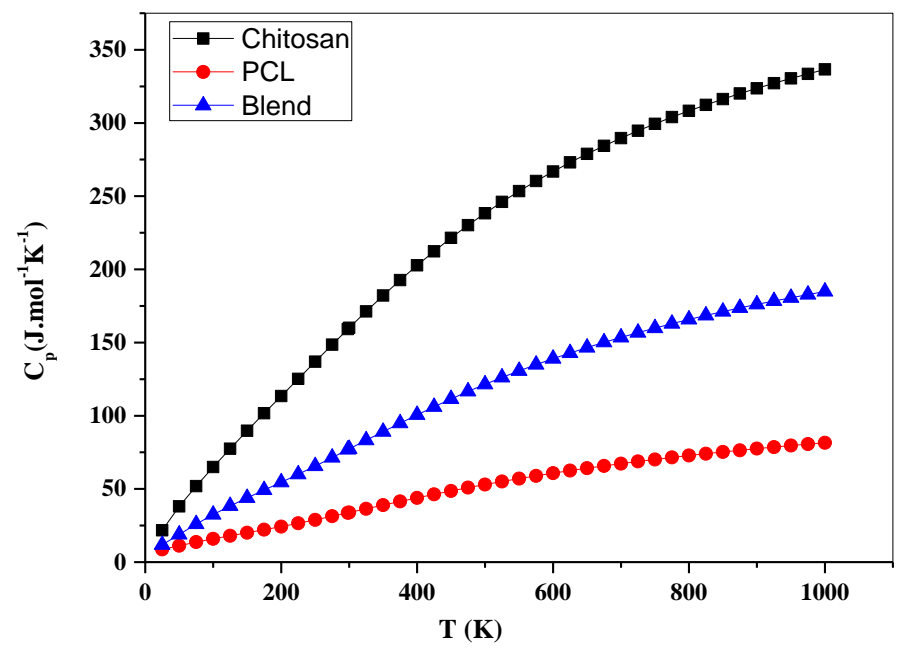

Figure (3) temperature versus heat capacity of the studied samples

Figure (4) shows the relation between temperature and free energy of studied samples. The free energies decreases with increasing temperature for all studied samples and the most probable reason for this behavior may attributed to mixing effect. 


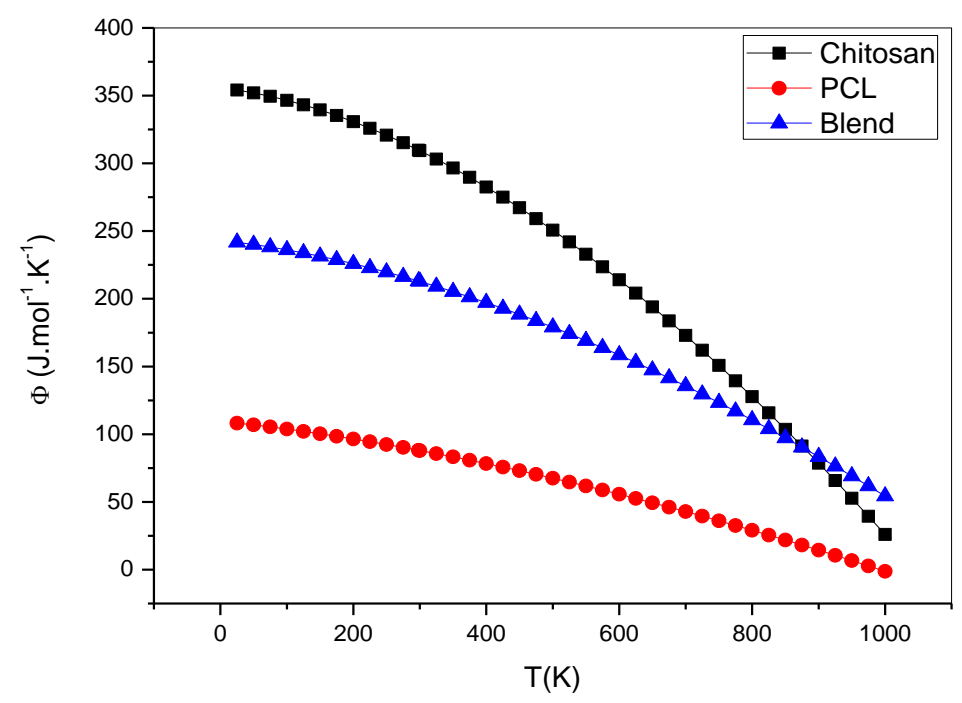

Figure (4) temperature versus free energy of the studied samples

Figure (5) represent the relation between the temperature and the enthalpy of studied samples.

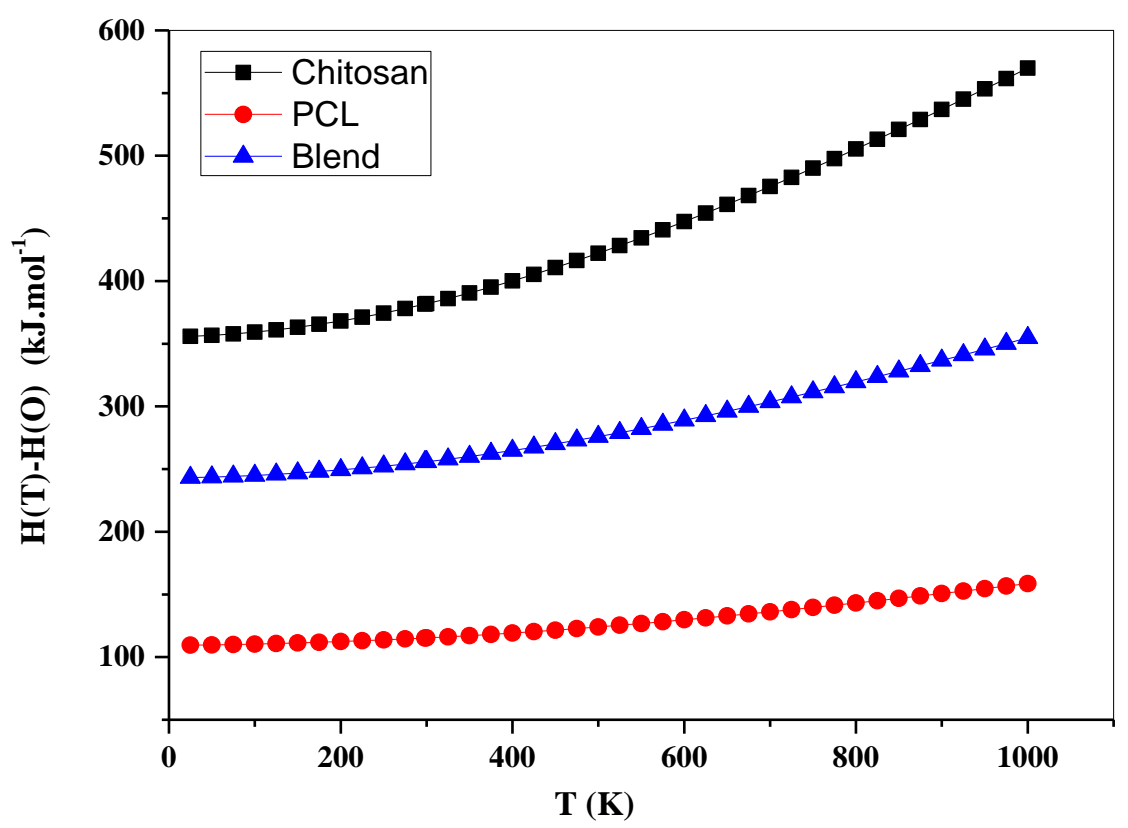

Figure (5) temperature versus enthalpy of the studied samples 
Energy optimization of the selected samples shows a three dimensional arrangement of the constituting atoms per unit cell which results in absorption behavior correlated to the frequency previously studied [12, 13] and compared and supported with published data of similar system [14, 15]. Such compatibility open gate for the comparison of other related data which can be obtained by the same route including thermodynamic parameters such as specific heat, entropy, free energy and enthalpy even in a nonreachable temperatures. Obtained thermodynamic parameters shows some type of interaction and complexation indicated by the intermediate values for semi-natural blend of all parameters over the whole studied temperatures $(25-1000 \mathrm{~K})$ with a comparable values at marked room temperature $(289.15 \mathrm{~K})$ in all graphs and tables. In addition, the absence of negative or imaginary frequencies in calculated data point out and support the structural arrangement of both virgin polymers and also in their suggested complexes poly blend. In the contrary of figures (2,3 and 4) which show convex behavior according to equation (2), the enthalpy shows a concave behavior eq. (1).

\section{Conclusions}

Calculated vibrational spectra proved the equilibrium of suggested structures especially in the absence of imaginary frequencies. Present study indicates that the use of DFT can predict the thermodynamic parameters at temperatures not reached through any experimental methods. Obtained data of polymer blend lay between that of both polymeric constituents indicating interaction and complexation of polymer blend at all temperatures. The plot of the entropy and heat capacity as a function of temperature show a convex behavior while the enthalpy show a concave behavior supported by the given equations.

\section{Conflicts of Interest}

No conflict of interest.

\section{Funding Statement}

The authors received no direct funding for this research.

\section{References}

1- Tambara M, Striccoli M., Comparalli R, Curri MI, Patralla A, ad Agodtiano A, Optical Properties of Hybird Composites, based on highly luminescent CDS Nanocrystals in Polymer, Nanotechnology 2004;15(4):S240.

2- Hussain F, Hajjati M, Okamato M, Gorga RE, Polymer Matrix: Nanocomposites, Processing Manufacturing and Applications: an Overview, J. of Composite Materials 2006;40(17)1511.

3- Khor E, Lim LY, Implantable applications of chitin and chitosan, Biomaterials 2003;24(13):2339.

4- Pawlak A, Mucha M, Thermogravimetric and FTIR studies of chitosan blends, Thermochimica Acta 2003;396(1-2):153.

5- Shahidi F, Arachchi JKV, Jeon YJ, Food applications of chitin and chitosans, Trends in Food Science \& Technology 1999;10(2):37.

6- Davies RC, Neuberge A, Wilson BM, Dependence of Lysozyme Activity on PH and Ionic Strength, Biochimica Et Biophysica Acta 1969;178(2):294.

7- Pangburn S H, Trescony PV, Heller J, Lysozyme Degradation of Partially Deacetylated Chitin, Its Films and Hydrogels, Biomaterials 1982;3(2):105.

8- Shigemasa $Y$, Saito $K$, Sashiwa H, Saimoto H, Enzymatic Degradation of Chitins and Partially Deacetylated Chitins, International Journal of Biological Macromolecules, 1994;16(1):43.

9- Wiseman DM, Domb AJ, Kost J, Handbook of Biodegradable Polymers, Taylor \& Francis, 1998. 
10- Santin M, Huang S, Iannace S, Ambrosio L, Nicolais L, Peluso G, Synthesis and characterization of a new interpenetrated poly(2- hydroxyethylmethacrylate)-gelatin composite polymer, Biomaterials 1996; 17(15):1459.

11- Olabarrieta I, Forsstrom D, Gedde UW, Hedenqvist MS, Transport properties of chitosan and whey blended with poly(epsilon-caprolactone) assessed by standard permeability measurements and microcalorimetry, Polymer 2001;42(9):4401.

12- Vijayalekshmi V, Khastgir D, Chitosan/partially sulfonated poly (vinylidene fluoride) blends as polymer electrolyte membranes for direct methanol fuel cell applications, Cellulose 2018;25(1):661.

13- Demetgül C, Beyazit N, Synthesis, characterization and antioxidant activity of chitosan-chromone derivatives, Carbohydrate polymers 2018;181:812.

14- Correlo VM, Boesel LF, Bhattachary M, Mano JF, Neves NM, Reis RL, Properties of melt processed chitosan and aliphatic polyester blends, Materials Science and Engineering: A 2005; 403(1-2)57.

15- SarasamA, Madihally SV, Characterization of chitosan-polycaprolactone blends for tissue engineering applications, Biomaterials 2005; 26(27):5500. 